\title{
Article \\ Nicotinamide Effectively Suppresses Fusarium Head Blight in Wheat Plants
}

\author{
Yasir Sidiq ${ }^{1,2}{ }^{\mathbb{D}}$, Masataka Nakano ${ }^{3}$, Yumi Mori ${ }^{3}$, Takashi Yaeno ${ }^{4}$, Makoto Kimura ${ }^{5}$ \\ and Takumi Nishiuchi $1,3, *$ (D)
}

1 Division of Life Science, Graduate School of Natural Science and Technology, Kanazawa University, Kanazawa 920-1192, Japan; ys120@ums.ac.id

2 Biology Education, Faculty of Teacher Training and Education, Universitas Muhammadiyah Surakarta, Sukoharjo 57162, Indonesia

3 Institute for Gene Research, Advanced Science Research Center, Kanazawa University, Kanazawa 920-8640, Japan; masa4nak@gmail.com (M.N.); moriyumi0122@gmail.com (Y.M.)

4 Department of Agriculture, Ehime University, Ehime 790-8566, Japan; yaeno@agr.ehime-u.ac.jp

5 Division of Molecular and Cellular Biology, Graduate School of Bioagricultural Sciences, Nagoya University, Nagoya 464-8601, Japan; mkimura@agr.nagoya-u.ac.jp

* Correspondence: tnish9@staff.kanazawa-u.ac.jp; Tel.: +81-76-265-2772; Fax: +81-76-265-2774

Citation: Sidiq, Y.; Nakano, M.; Mori, Y.; Yaeno, T.; Kimura, M.; Nishiuchi, T. Nicotinamide Effectively Suppresses Fusarium Head Blight in Wheat Plants. Int. J. Mol. Sci. 2021, 22, 2968. https://doi.org/10.3390/ijms22062968

Academic Editor: Luigi Cattivelli

Received: 14 February 2021

Accepted: 5 March 2021

Published: 15 March 2021

Publisher's Note: MDPI stays neutral with regard to jurisdictional claims in published maps and institutional affiliations.

Copyright: (C) 2021 by the authors. Licensee MDPI, Basel, Switzerland. This article is an open access article distributed under the terms and conditions of the Creative Commons Attribution (CC BY) license (https:// creativecommons.org/licenses/by/ $4.0 /)$.

\begin{abstract}
Pyridine nucleotides such as a nicotinamide adenine dinucleotide (NAD) are known as plant defense activators. We previously reported that nicotinamide mononucleotide (NMN) enhanced disease resistance against fungal pathogen Fusarium graminearum in barley and Arabidopsis. In this study, we reveal that the pretreatment of nicotinamide (NIM), which does not contain nucleotides, effectively suppresses disease development of Fusarium Head Blight (FHB) in wheat plants. Correspondingly, deoxynivalenol (DON) mycotoxin accumulation was also significantly decreased by NIM pretreatment. A metabolome analysis showed that several antioxidant and antifungal compounds such as trigonelline were significantly accumulated in the NIM-pretreated spikes after inoculation of F. graminearum. In addition, some metabolites involved in the DNA hypomethylation were accumulated in the NIM-pretreated spikes. On the other hand, fungal metabolites DON and ergosterol peroxide were significantly reduced by the NIM pretreatment. Since NIM is relative stable and inexpensive compared with NMN and NAD, it may be more useful for the control of symptoms of FHB and DON accumulation in wheat and other crops.
\end{abstract}

Keywords: nicotinamide; pyridine nucleotide; fusarium head blight; trichothecene mycotoxin; DNA hypomethylation; antifungal compound; metabolomics

\section{Introduction}

Plants defend themselves against various pathogens by both of chemical and physical defense systems [1]. Antimicrobial compounds which accumulate due to pathogen challenge are known as phytoalexins [2]. However, phytopathogens often overcome plant chemical defenses and enter the plant tissue. Therefore, biochemicals such as pesticides and fungicides are useful for the control of crop diseases [3,4]. However, the foods derived from agrochemical-contaminated crops often present health hazards to humans and domestic animals [5]. In addition, fungicide- and pesticide-resistant pathogen strains have been widely reported [6]. Plant defense activators induce the plant's immune response. Probenazole is one such activator and can enhance resistance against rice blast fungus and other diseases [7,8]. Additionally, both 2,6-dichloroisonicotinic acid (INA) and benzo $(1,2,3)$ thiadiazole-7-carbothionic acid S-methyl ester (BTH) are SA analogues which activate the systemic acquired resistance (SAR) signal transduction pathway [9,10].

Among the various phytopathogens, certain fungal pathogens produce toxic secondary metabolites (mycotoxins) which are harmful to humans and domestic animals [11,12]. Fusarium species such as Fusarium graminearum infect the flowers of wheat and barley 
spikes, and most of them can produce trichothecene mycotoxins $[13,14]$. These fusarium diseases are called Fusarium head blight (FHB). Commercial cultivars showing strong FHB resistance are not available in wheat and barley [15]. Therefore, fungicides are usually sprayed multiple times on their flowers [16]. However, pesticide-contaminated grains are also toxic to humans and animals. Therefore, in the present research, we attempt to identify useful natural products for the control of Fusarium head blight in cereal crops. In a previous study, we found that nicotinamide mononucleotide (NMN), a precursor of nicotinamide adenine dinucleotide (NAD), was highly accumulated in FHB-resistant barley cultivars [17]. We also revealed that NMN acted as a plant defense activator in Arabidopsis. Furthermore, the application of NMN was shown to enhance disease resistance against $F$. graminearum and suppress deoxynivalenol (DON) mycotoxin accumulation in barley [17]. We also found that L-Thr can suppress trichothecene biosynthesis in F. graminearum [18]. Thus, these metabolites may be useful for the control of disease injury and mycotoxin reduction in cereals.

Recently, extracellular nicotinamide adenine dinucleotide (eNAD) was shown to be able to activate plant immune response by binding to plant receptors $[19,20]$. Therefore, increasing the eNAD content was found to be effective in suppressing the disease resistance of Arabidopsis to Pseudomonas syringae pv. maculicola ES4326 (Psm ES4326) [21]. As stated above, NMN also acts as a plant defense activator and enhances FHB disease resistance in Arabidopsis and barley [17]. Since NAD and NMN contain the nucleotide, they are relatively unstable and expensive to produce. Among these derivatives, nicotinamide, which is stable and cheap, has antioxidant activity [22]. Therefore, herein, we examine the effects of nicotinamide on the suppression of FHB in wheat plants. For this purpose, we use a dwarf model wheat cultivar USU-Apogee that is susceptible to FHB. We reveal that the application of nicotinamide is effective in suppressing FHB in wheat plants. A metabolome analysis suggested that many defense-related metabolites were likely involved in the NIM-induced FHB resistance.

\section{Results}

\subsection{Disease Development of FHB in the Wheat Model Cultivar}

Since the USU-Apogee is a dwarf wheat cultivar with a short life cycle, this cultivar is useful for the study of FHB in wheat [23]. USU-apogee revealed the FHB susceptible phenotype, which is similar to that of the known cultivar, Wheaton [23]. Therefore, we used the USU-apogee to evaluate the effects of NIM and NMN on FHB resistance. We monitored the spread of disease on the wheat spike after inoculation of F. graminearum. Fungal conidia were sprayed onto spikes with open flowers, and then inoculated spikes were kept in high humidity for two days. The disease symptoms appeared on the wheat spikes at 3 days postinoculation (dpi) in the control treatment. Figure $1 \mathrm{a}, \mathrm{b}$ show that the symptoms of FHB disease became visible at $3 \mathrm{dpi}$ in the flowerets of the control inoculated spikes. As shown in Figure 1a, the rate of incidence at 3 dpi was only $5 \%$, but this gradually increased to $10 \%$ at $5 \mathrm{dpi}$ in the control treatment. From 5 to $7 \mathrm{dpi}$, the rates of disease incidence increased rapidly to about $26 \%$ in the control inoculated spikes. As shown in Figure $1 \mathrm{~b}$, severe symptoms were observed only at $7 \mathrm{dpi}$ in control spikes.

\subsection{Nicotinamide Pretreatment Enhanced the FHB Resistance in Wheat Plants}

In this study, we examined whether NMN and related metabolite NIM were effective in suppressing the disease symptoms of FHB and DON accumulation in wheat spikes. NIM does not contain any nucleotides, and is stable at room temperature. In addition, the cost of NIM is significantly less than those of NMN and NAD. For this purpose, solutions of NMN or NIM were sprayed onto the wheat spikes prior to inoculation of $F$. graminearum. As shown in Figure 1a, the incidence rates of inoculated spikes by NMNand NIM-pretreatment decreased at $3 \mathrm{dpi}$ in the water-pretreated control spikes. This fact indicated that plant immune response had already been activated by NMN- and NIMpretreatment in the early stage of infection. Disease symptoms in the inoculated spikes 
after pretreatment with NMN and NIM gradually developed, and finally reached about $12 \%$ and $8 \%$, respectively, at $7 \mathrm{dpi}$ (Figure $1 \mathrm{a}, \mathrm{b}$ ). As stated above, the incidence rate in the water-pretreated spikes was about $26 \%$. Significant differences of disease incidence were observed in NMN- and NIM-pretreated spikes compared with water-pretreated spikes; see Figure 1a. This result clearly showed that both NMN and NIM are capable of inducing plant defense response to suppress the development of FHB in wheat spikes. As previously reported, NMN and NAD are effective at decreasing the symptoms of FHB disease in barley and Arabidopsis. In addition, NMN and NIM application was also found to be useful in controlling FHB disease in wheat plants. As stated above, although NIM did not contain any nucleotides, significant suppression of FHB symptoms was observed in NIM-pretreated wheat spikes. Since NIM has higher stability with lower cost compared to pyridine nucleotides, NMN and NAD, it is potentially a more useful candidate for the control of FHB.

(a)

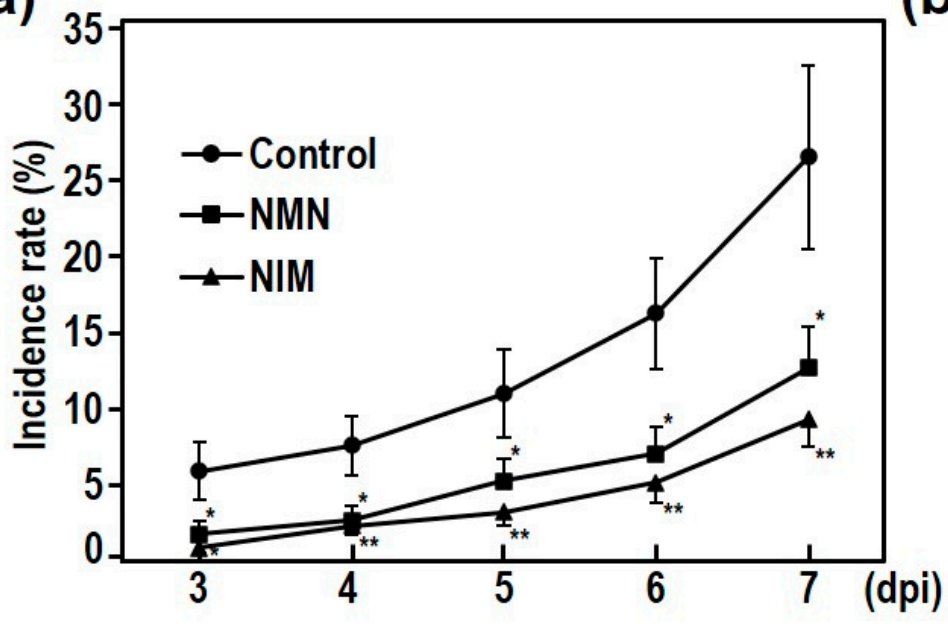

(b)

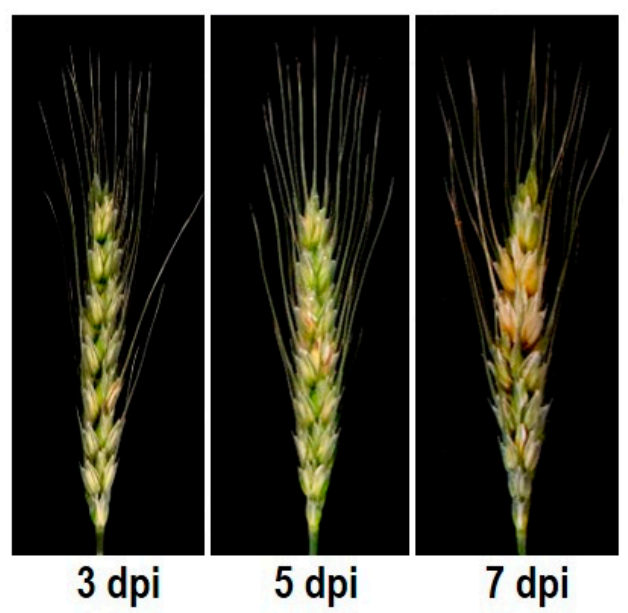

Figure 1. Disease incidence of Fusarium head blight (FHB) gradually increased from 3 dpi to 7 dpi. (a) The incidence of FHB disease on spikes of wheat cultivar USU-Apogee from 3 to $7 \mathrm{dpi}$. These spikes were sprayed with water, NMN, or NIM before inoculation. Each bar represents standard error $(n=12)$. Results of student's t-test are shown: ${ }^{*} p<0.05,{ }^{* *} p<0.01$. (b) Representative photographs of symptom development in the water-treated control spikes at 3, 5, and $7 \mathrm{dpi}$.

To further analyze the effects of NMN and NIM in the control of FHB, fungal gDNA and DON accumulation in wheat spikes were measured. The DON accumulation was sometimes not correlated with the severity of disease symptoms in wheat and barley [24]. Therefore, the measurement of DON accumulation was necessary to evaluate the efficacy of the FHB control agents. Figure 2a shows that NMN and NIM pretreatment effectively suppressed the disease symptoms at $7 \mathrm{dpi}$ in wheat spikes. The ratio of the fungal gDNA to the total gDNA in the control spikes was about $0.13 \%$; however, those were apparently decreased to $0.06 \%$ and $0.02 \%$ in NMN- and NIM-pretreated wheat spikes, respectively; see Figure $2 \mathrm{~b}$. This result indicated that the NMN and NIM pretreatment effectively suppressed the propagation of cells of $F$. graminearum in wheat spikes. In the control, DON accumulated at more than $2 \mathrm{ppm}$ at $7 \mathrm{dpi}$ in the inoculated spikes; see Figure 2c. In contrast, the NMN and NIM-pretreated spike exhibited the about $1 \mathrm{ppm}$ and $0.35 \mathrm{ppm}$ DON accumulation, respectively. Thus, the propagation of pathogenic cells and DON accumulation were reduced to one-sixth and one-fifth by NIM pretreatment. The effects of NMN were similar, but slightly weaker, compared with NIM pretreatment. As mentioned, both NMN and NIM pretreatment effectively suppressed the initial infections by $3 \mathrm{dpi}$; see Figure 1a. Then, the increasing rates of disease incidence in the NMN- and NIM-pretreated spikes were also lower than those of the water-pretreated control spikes at 3 and $7 \mathrm{dpi}$. Therefore, the initial infection and development of FHB disease were effectively suppressed by NMN and NIM application. Long term incubation after inoculation is important for 
the evaluation of FHB severity. Therefore, we examined the incidence rates of FHB disease from 4 to $28 \mathrm{dpi}$ in our inoculation system; see Figure S1. In our experimental condition, the incidence rates reached about $100 \%$ at $12 \mathrm{dpi}$. However, the effects of NIM and NMN were still observed at 12 dpi (Figure S2).

(a)

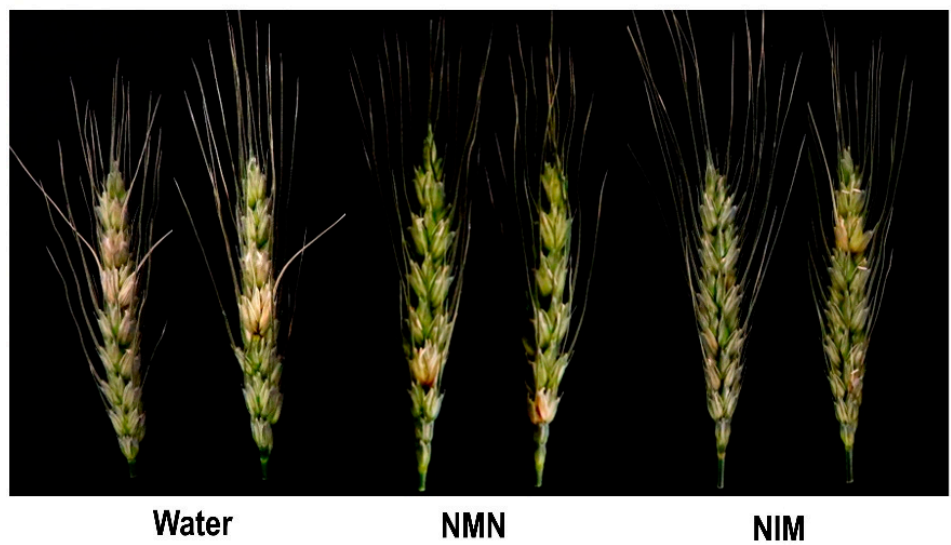

(b)

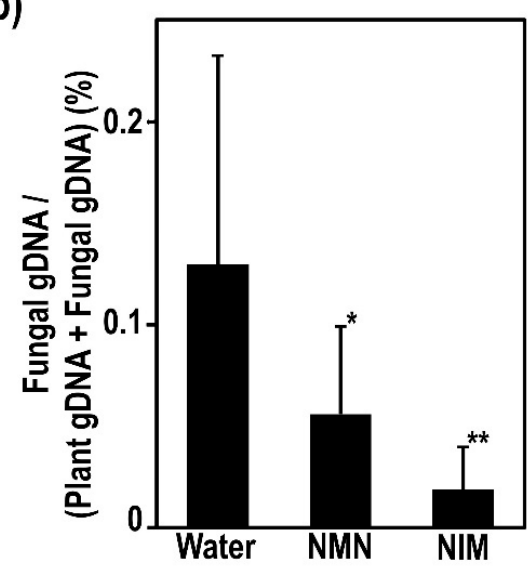

(c)

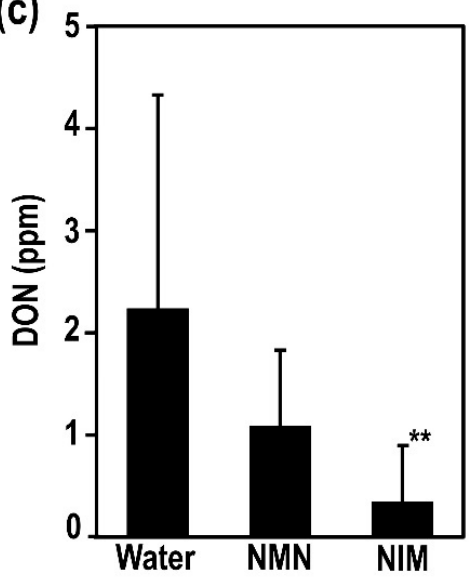

Figure 2. Nicotinamide mononucleotide (NMN) and nicotinamide (NIM) pretreatment enhanced disease resistance against F. graminearum in wheat spikes. NMN or NIM was sprayed onto spikes of susceptible cultivar USU-Apogee before inoculation. A conidia solution with $1 \times 10^{4}$ conidia/mL was applied to these spikes by spraying. (a) The representative photographs of inoculated spikes at $7 \mathrm{dpi}$ after water, NMN or NIM pretreatment. (b) The ratio of the fungal gDNA to the total gDNA in the water-, NMN-, NIM-pretreated spikes were quantified by qPCR, (c) DON accumulations of water-, NMN-, and NIM-pretreated spikes were measured. Each bar represents standard deviation $(n=12)$. Student's $t$-test: ${ }^{*} p<0.05,{ }^{* *} p<0.01$.

Furthermore, we examined the effects of NMN and NIM on two other Japanese cultivars; see Figures S3 and S4. It is known that the FHB resistance of Harukirari and Haruyutaka are intermediate and weak, respectively, among Japanese varieties [25,26]. The effects of NIM and NMN were also observed in two cultivars, although different degrees of FHB resistance were observed among the two cultivars (Figures S3 and S4). These results suggested that NIM and NMN may be useful to control FHB disease in many wheat cultivars.

\subsection{Effects of NMN and NIM Pretreatment on Pyridine Metabolites of Wheat Inoculated Spikes}

As mentioned above, disease symptoms and DON accumulations were suppressed by NIM and NMN pretreatment in wheat spikes. To examine the effects of these metabolites, we investigated the amounts of NMN, NIM and related metabolites using LC-MS/MS. We extracted the metabolites from inoculated spikes at $7 \mathrm{dpi}$ with water, NMN, and NIM 
pretreatment. These extracted solutions were separated by UPLC and analyzed by MS spectrometer (Orbitrap QE plus). As shown in Figure 3, we measured pyridine nucleotides and related metabolites in these samples. The content of NMN was still significantly accumulated in the inoculated spikes after seven days of NMN spraying. In contrast, the NMN content was very low in the NIM- and water-pretreated leaves. This result showed that NMN was still present after seven days of spraying and likely affected the defense responses of wheat spikes. The contents of NIM and trigonelline (TRG) were slightly elevated in the NMN-pretreated spikes. The NAD and nicotinic acid (NA) contents in NMN-pretreated spikes were not different from those of the water-pretreated spikes. The apparent increase by NMN pretreatment was observed only in NMN content among five metabolites. NIM pretreatment caused a significant increase in not only NIM, but also TRG. Since TRG is a pyridine alkaloid which acts as an antimicrobial compound, accumulation of TRG likely contributed the enhanced FHB resistance in wheat spikes.<smiles>NC(=O)c1cccnc1</smiles>

NIM

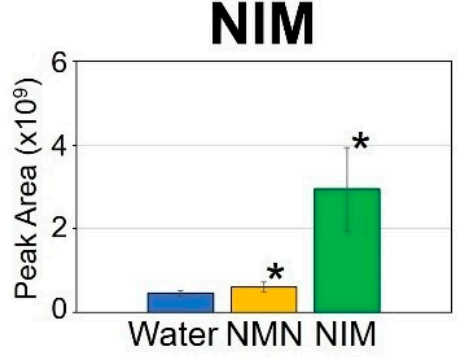<smiles>O=C(O)c1cccnc1</smiles>

NA

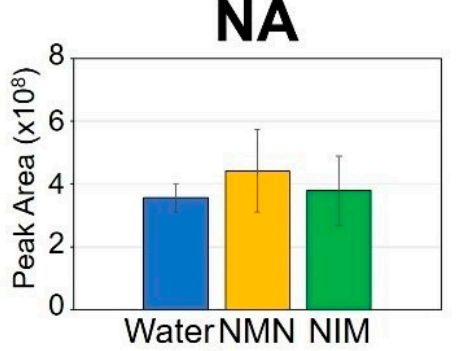<smiles>C[n+]1cccc(C(=O)[O-])c1</smiles>

TRG

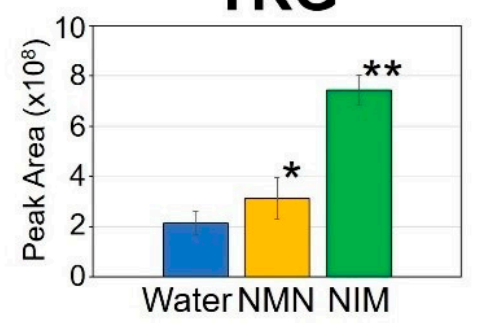<smiles>NC(=O)c1ccc[n+]([C@@H]2O[C@H](COP(=O)([O-])O)[C@@H](O)[C@H]2O)c1</smiles>

NMN

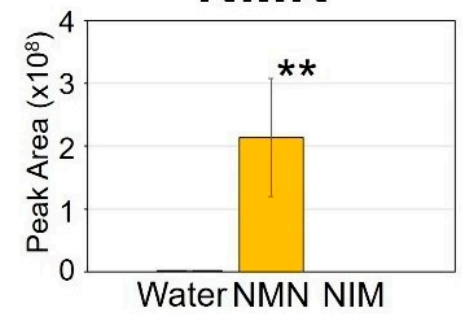<smiles>NC(=O)c1ccc[n+]([C@@H]2O[C@H](COP(=O)(O)OP(=O)(O)OC[C@H]3O[C@@H](n4cnc5c(N)ncnc54)[C@H](O)[C@@H]3O)[C@@H](O)C2O)c1</smiles>

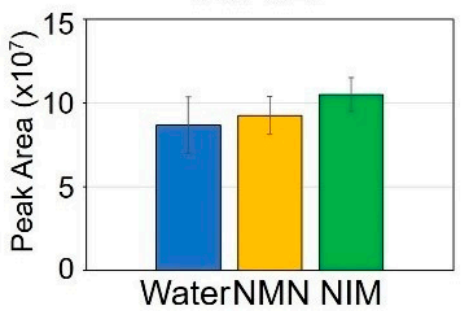

Figure 3. Effects of nicotinamide mononucleotide (NMN) and nicotinamide (NIM) pretreatment on pyridine metabolites in the wheat inoculated spikes. Relative amounts of metabolites NMN, NIM, nicotinic acid (NA), trigonelline (TRG), and nicotinamide adenine dinucleotide $\left(\mathrm{NAD}^{+}\right)$were measured in water-, NMN-, and NIM-pretreated spikes based on the peak area of their precursor ions. Each bar represents standard deviation. Student's $t$-test: ${ }^{*} p<0.05,{ }^{* *} p<0.01, n=4-5$. 


\subsection{Differentially Accumulated Metabolites in the NMN-and NIM-Pretreated Spikes}

We also performed comparative metabolome studies on water-, NMN-, and NIMpretreated spikes at $7 \mathrm{dpi}$. Many differentially accumulated metabolites were identified by LC-MS/MS analysis. In Figure 4, volcano plots show differentially accumulated metabolites by NMN and NIM pretreatment. These metabolites contained unspecified and redundant ones. The red area indicates upregulated metabolites due to NMN or NIM pretreatments (fold change $>1.5$ and $p$ value $<0.05$ ); the green area shows downregulated metabolites due to NMN or NIM pretreatment (Fold change $<0.67$ and $p$ value $<0.05$ ). It was found that 99 and 486 metabolites were up- and down-regulated, respectively, by NMN pretreatment in inoculated spikes at 7dpi. On the other hand, NIM pretreatment induced and reduced the contents of 375 and 1174 metabolites, respectively, in inoculated spikes. Thus, the effects of NIM pretreatment on the metabolite profile were apparently greater than those of NMN pretreatment.

(a)

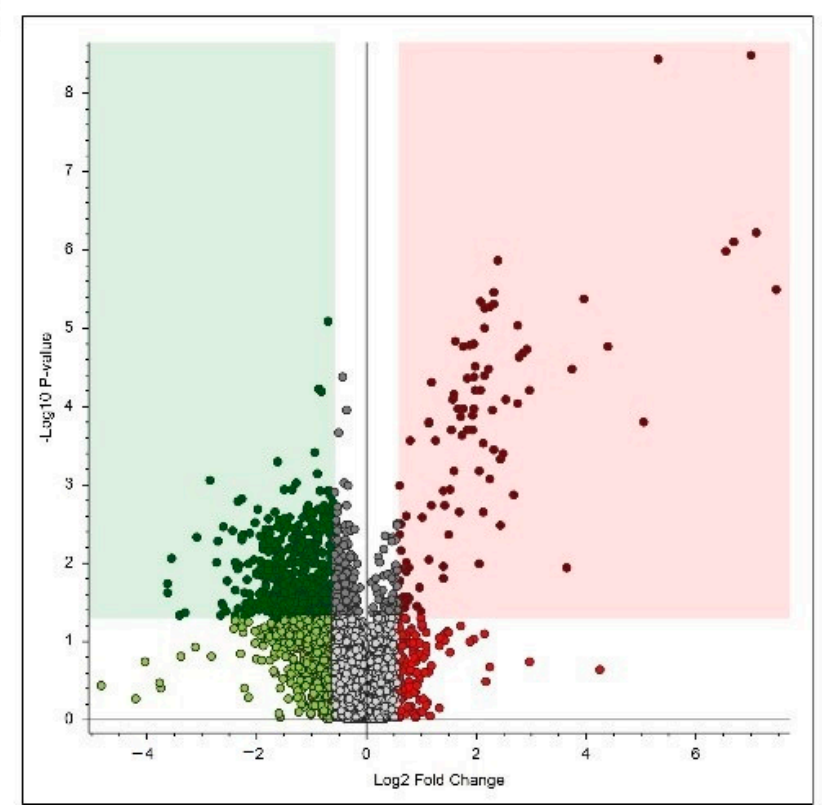

(b)

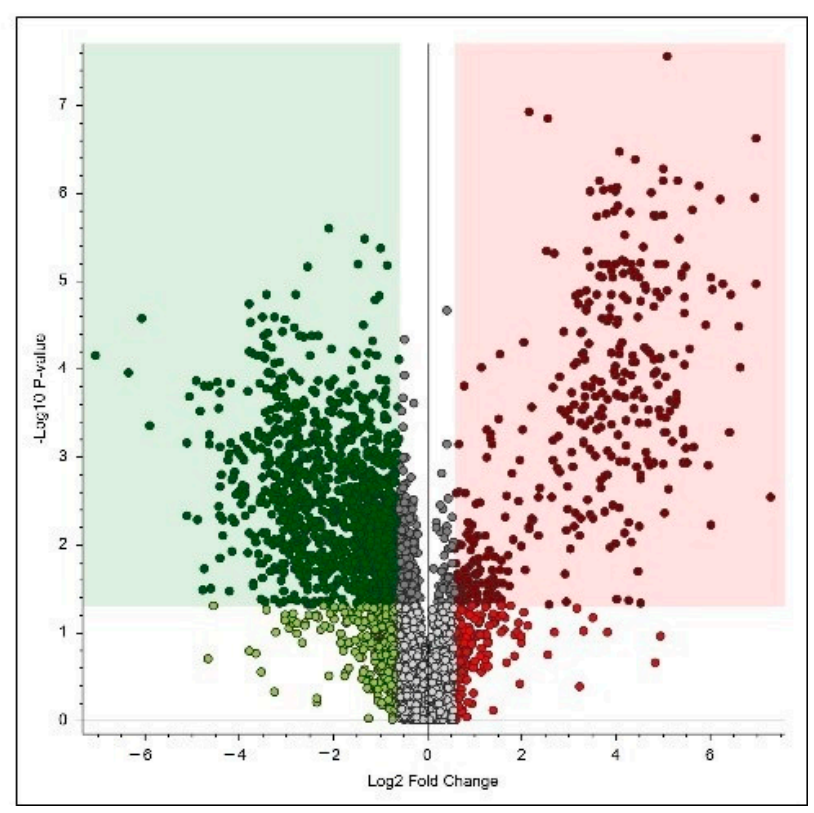

Figure 4. Volcano plots illustrate the distributions of quantified metabolites in the nicotinamide mononucleotide (NMN)and nicotinamide (NIM)-pretreated spikes at $7 \mathrm{dpi}$. The results of (a) NMN- and (b) NIM-pretreated spikes are shown. The vertical-axis is the $-\log 10$ of the $p$-value and the horizontal-axis is $\log 2$ of fold change of NMN- or NIM-pretreated spikes compared to the control ones. Cutoff values of fold change and $p$-value were 1.5 and 0.05 , respectively. The dots indicate detected metabolites containing the unidentified and redundant ones. Red squares indicate significantly accumulated metabolites, while green squares show significantly reduced metabolites.

Next, we checked the properties of each differentially regulated metabolite by NMN or NIM pretreatment. These metabolites were classified into three groups: (a) highly accumulated in the NIM-treated spikes; (b) highly accumulated in the NMN-pretreated spikes; and (c) reduced metabolites in the NMN- and NIM-pretreated spikes. Figure 5 shows the representative metabolites in each group. Antibiotics, as noted in Figure 5a, were highly accumulated in the NIM-pretreated spikes after inoculation of F. graminearum. Bacancosin is a plant saponin, i.e., a natural detergent which is harmful to the membranes of microbials [27]. Both debromohymenialdisine and buchananine are classified as alkaloids, most of which have antimicrobial activities [28]. It has been reported that buchananine is an antifungal compound [29], although large variation was observed in its content in NIM-pretreated spikes. Sulfamethazine has also been reported as an antimicrobial compound [30]. On the other hand, cyclo-Dopa 5-O-glucoside may act as a ROS scavenger [31]. DIMBOA-glucoside is likely related to defense signaling [32]. As shown in Figure 5b, 
cystothiazole A and picolinic acid are specifically accumulated by NMN-pretreatment. It has been reported that cystothiazole A has antifungal activity to Phytophthora capsica [33]. The fold increase of picolinic acid content by NMN-pretreatment was not large, but was statistically significant. Picolinic acid is known as an inducer of plant defense response against Magnaporthe oryzae [34].

(a)
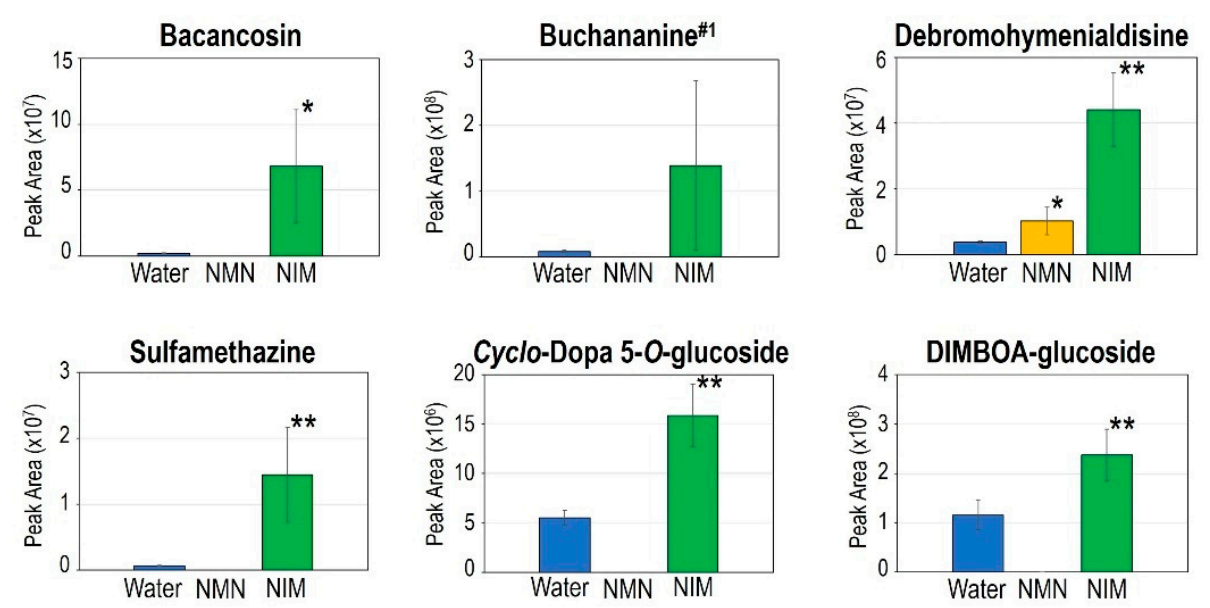

(b)
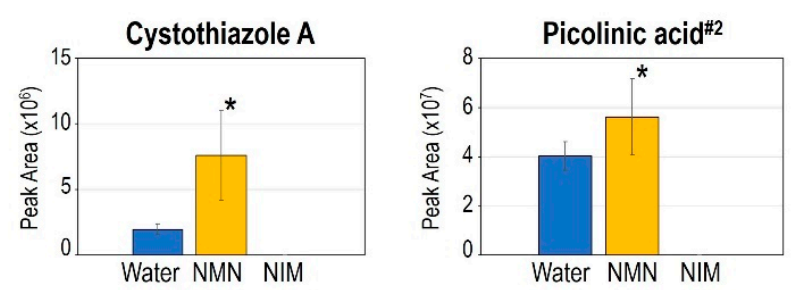

(c)
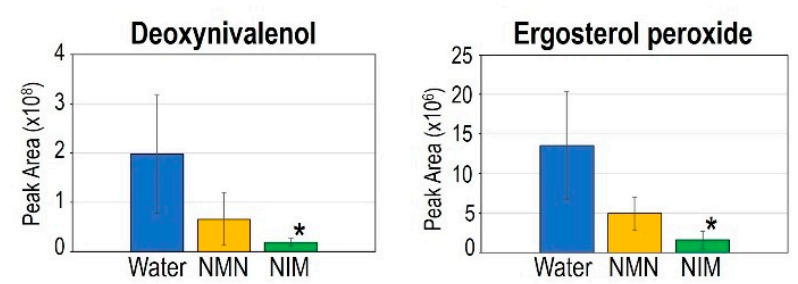

Figure 5. Representative differentially regulated metabolites by nicotinamide mononucleotide (NMN) and nicotinamide (NIM) pretreatment in inoculated spikes at 7 dpi. (a) Metabolites significantly accumulated by NIM pretreatment, (b) metabolites significantly accumulated by NMN pretreatment, and (c) metabolites significantly reduced by NIM pretreatment. The peak areas of the vertical axis show the abundance of each metabolite. (1) Buchananine was more than 1.5-fold more accumulated without statistical significance in NIM-pretreated spikes. (2) Picolinic acid was significantly accumulated in the NMN-pretreated spikes with a less than 1.5-fold change. Each bar represents one standard deviation $(n=4-5)$. Student's $t$-test: ${ }^{*} p<0.05,{ }^{* *} p<0.01$.

As shown in Figure 5c, two fungal metabolites are reduced by NMN- and NIMpretreatment. As stated above, DON was significantly decreased in the NIM-pretreated spikes. Correspondingly, derivatives of ergosterol, which is specifically found in the fungal membrane, were also shown to be significantly decreased in the NIM-pretreated spikes. These results also support the hypothesis that FHB disease and mycotoxin accumulation can be significantly suppressed by NIM pretreatment. 


\section{Discussion}

Many natural products have been put forward as candidates to control FHB resistance and mycotoxin accumulation in wheat and barley. Some antifungal compounds have been proposed as potential FHB control agents. The plant alkaloid antofine was shown to have antifungal activity, and significantly suppressed the development of $F$. graminearum at 150 $\mathrm{ug} / \mathrm{mL}$ concentration in wheat spikes [35]. In this study, we also identified three alkaloids, TRG, buchananine, and debromohymenialdisine, that were highly accumulated in the NIM-pretreated spikes in comparison with control spikes. Since both TRG and buchanaine are pyridine alkaloids, the application of NIM likely contributed to their accumulation. It has been reported that high nitrogen causes TRG accumulation in tomato leaves and enhances resistance against the fungal pathogen, Fusarium oxysporum [36]. In addition, the application of TRG to the leaves of barley plants reduced disease symptoms due to powdery mildew up to $56 \%$ [37]. Buchananine was shown to possess broad antibacterial activity against eight out of the ten bacteria species examined [38]. Therefore, these metabolites likely play roles in FHB resistance in wheat plants.

Debromohymenialdisine was reported as an effective insecticidal compounds [39]. As stated above, bacancosin is a plant saponin which is harmful to microbial membranes [27]. Sulfamethazine also has antimicrobial activities [30]. Thus, the accumulation of these antimicrobial compounds in NIM likely plays an important role in enhancing FHB disease resistance in wheat spikes.

It is known that NIM has antioxidant activity relative to abiotic stress in plants [22,40]. Similarly, we previously reported that NMN pretreatment decreased the ROS accumulation in Arabidopsis leaves inoculated with F. graminearum [17]. On the other hand, DNA hypomethylation effects by NIM have been reported in plant cells [22]. It has also been reported that TRG application reduces DNA methylation in barley and enhances disease resistance to powdery mildew [37]. In general, DNA hypomethylation is related to the activation of gene expression [41]. Furthermore, sulfamethazine, which is a pyrimidine, suppresses epigenetic silencing through DNA methylation [42]. Thus, these metabolites, including NIM itself, likely cause DNA hypomethylation and the activation of immune response genes. DIMBOA-glucoside has been reported as an anti-insect compound in plants [43]. In addition, DIMBOA-glucoside was found to be accumulated in the MPK6overexpressed of Zea mays [44], suggesting that production of DIMBOA-glucoside was regulated by the MAPK-dependent defense signaling pathway. Interestingly, it has been reported that DIMBOA suppressed the expression of trichothecene biosynthetic genes in F. graminearum [45]. Mycotoxin production was shown to be significantly suppressed without disturbing fungal growth [45]. In this study, we did not detect DIMBOA in all wheat samples (data not shown). Thus, NIM and other metabolites have antioxidant and DNA hypomethylation activities, and these activities were likely involved in the observed enhanced FHB resistance in wheat spikes. However, we should experimentally confirm the occurrence of hypomethylation by NIM treatment in a future study.

As shown in Figure 5b, two metabolites were specifically accumulated in the NMNpretreated spikes. Cystothiazole A has antifungal activity against a broad range of microorganisms including Aspergillus fumigatus, Botritys cinerea and Phytophora capsici [33]. Cystothiazole A is a bithiazole which inhibits respiration, since it interferes with NADH oxidation [33]. The application of picolinic acid at low concentrations stimulates antioxidative response, resulting in a decrease of blast symptoms in rice [34]. Therefore, these metabolites are at least partially involved in FHB resistance due to NMN.

We also identified two fungal metabolites which were significantly decreased in the NIM-pretreated spikes (Figure 5c). Such differences were also observed in the NMNpretreated spikes, but there were not significant. A significant decrease in DON accumulation was confirmed in NIM-pretreated spikes by LC-MS/MS analysis (Figures $2 c$ and $5 c$ ). Ergosterol peroxide is a derivative of ergosterol that is specifically observed in the membrane of fungal cells [46]. A significant reduction of ergosterol peroxide was observed in the NIM-pretreated spikes, indicating that the amounts of fungal cells therein were 
apparently decreased (Figure 5c). This result is consistent with the quantification of fungal gDNA (Figure 2c). Taken together, NIM pretreatment effectively suppressed the progression of fungal cells and the production of mycotoxin in inoculated spikes. Our identified metabolites support the hypothesis that NIM is a useful candidate to control FHB disease in wheat and other crops.

Other natural FHB control agents have been reported. For example, tannic acid is a plant polyphenol which inhibits conidia germination and mycelium growth of $F$. graminearum [47]. The application of tannic acid was found to reduce disease severity and DON in spikes of wheat cultivar USU-Apogee [47]. Similar effects were observed in a field test with artificial inoculation of F. graminearum [47]. Recently, it was reported that the application of chitosan hydrochloride derived from chitin suppressed the severity of FHB and DON accumulation in wheat spikes. In addition, chitosan treatment activated the SAR signaling pathway and caused induction of some defense genes in wheat spikes [48]. Additionally, some natural products have been identified as inhibitors of trichothecene biosynthesis in F. graminearum. The glutamine analogue, acivicin, suppressed the expression of Tri4, Tri5, and Tri6 genes and reduced mycotoxin production in the medium [49]. In addition, the amino acid L-Thr also suppressed trichothecene mycotoxin production in the medium and host plants [18]. Since these inhibitors of trichothecene biosynthesis exhibited different effects compared to NIM and NMN, additive effects can be expected, which may be useful for controlling FHB disease symptoms and mycotoxin accumulation in wheat spikes. Therefore, we will perform field inoculation tests using such mixtures in the near future.

\section{Materials and Methods}

\subsection{Plant Material and Growth Conditions}

Wheat plants (Triticum aestivum) cultivar USU-Apogee were used in this study [23]. The seeds were sown on filter paper supplied with water for two days in constant darkness at $22{ }^{\circ} \mathrm{C}$. The germinated seedlings were then transferred to soil and grown in a growth

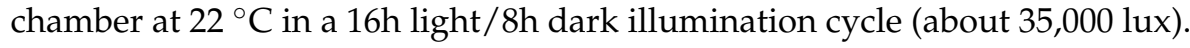

\subsection{Pretreatment of Compounds and Fungal Inoculations}

Fusarium graminearum strain $\mathrm{H} 3$ was used for the inoculation assay [17]. Fungal stocks were cultured on a potato dextrose agar (PDA) plate and stored at $4{ }^{\circ} \mathrm{C}$. We prepared the conidia solution as previously described [50].

Pretreatments of chemicals were performed three days and $4 \mathrm{~h}$ before inoculation, as described previously [17]. NMN (Chombi-block Inc., San Diego, USA) and NIM (TCI Co., Ltd., Tokyo, Japan) was dissolved with sterile distilled water at a concentration of $10 \mathrm{mg} / \mathrm{mL}(10,000 \mathrm{ppm})$ and kept at $-20^{\circ} \mathrm{C}$. The working concentration was $500 \mathrm{ppm}$ with $0.01 \%(v / v)$ silwet L77. Twelve plants were used for each chemical treatment.

Spray inoculations with F. graminearum were performed using flowering wheat spikes. The concentration of conidia solution was adjusted to $1 \times 10^{4}$ conidia/mL in $1 \times$ PBS with $0.001 \%(v / v)$ silwet L77, and was then applied to wheat spikes by spraying [17]. Inoculated wheat spikes were placed in a container with water and covered using plastic wrap to maintain high humidity. Then, inoculated spikes were incubated at $22{ }^{\circ} \mathrm{C}$ with low light intensity. At $2 \mathrm{dpi}$, the plastic wraps were removed. The inoculated spikes were incubated for an additional five days (total seven days after inoculation).

\subsection{Evaluation of Disease Incidence Rates}

The effects of NMN and NIM on FHB resistance were evaluated by the rate of disease incidence in the inoculated wheat spikes. The rate of disease incidence showed the ratio of the diseased florets to total florets in each spike [23]. Brown or premature bleaching in the single floret was counted as evidence of disease [51]. 


\subsection{The Quantification of Fungal $g D N A$}

Inoculated wheat spikes were crushed to obtain a fine powder using stainless beads and shake master neo (Bio Med. Sci., Tokyo, Japan) at $1500 \mathrm{rpm}$ for $2 \mathrm{~min}$ with liquid nitrogen cooling. The genomic DNAs were isolated from about $70 \mathrm{mg}$ fine powder using a Maxwell RSC Purefood GMO and Authentication Kit (Promega, Madison, WI, USA) with Maxwell RSC instrument (Promega, Madison, WI, USA). The quantities of F. graminearum gDNA and wheat gDNA in inoculated spikes were determined by qPCR using a AriaMx Real-Time PCR system (Agilent Tech., Santa Clara, CA, USA) with the reagent of $2 \times$ Brilliant III Ultrafast SYBR Green (Agilent Tech., Cedar Creek, TX, USA). For the quantification of $F$. graminearum gDNA, the F. graminearum EF-1a gene was amplified [17]. To quantify wheat gDNA, T. aestivum $\alpha$-tubulin gene was amplified, as previously described [52].

\subsection{Quantification of DON Accumulation}

DON accumulation in wheat spikes was measured from $10 \mathrm{mg}$ of fine powders using QuickScan DON3 (Envirologix, Inc., Portland, ME, USA). Five hundred microliters of sterile water was added to the fined powders. This were then vortexed at room temperature for one minute. Supernatants of samples were collected by centrifugation (5000 rpm) at room temperature for $10 \mathrm{~min}$. The DON accumulation in $200 \mu \mathrm{L}$ supernatant was measured using Envirologix QuickScan System (Envirologix, Inc., Portland, ME, USA) following the manufacturer's instructions.

\subsection{Extraction of Metabolites}

The extraction of metabolites was conducted as previously described [53]. Briefly, $50 \mathrm{mg}$ samples were measured and resuspended by vortex with $250 \mu \mathrm{L}$ of precooled $80 \%$ $(v / v)$ methanol containing $0.1 \%(v / v)$ formic acid. The samples were chilled on ice for $5 \mathrm{~min}$ and then centrifuged at $15,000 \mathrm{rpm}$ for $5 \mathrm{~min}$ at $4{ }^{\circ} \mathrm{C}$. Two hundred microliters of the supernatants was transferred to new tubes and diluted to a $53 \%(v / v)$ methanol concentration using water. Subsequently, the samples were centrifuged at 15,000 rpm at $4{ }^{\circ} \mathrm{C}$ for $10 \mathrm{~min}$. The supernatants were transferred to vials for UHPLC.

\subsection{UHPLC-MS/MS Analysis}

An Ultimate 3000 UHPLC system (Thermo Fisher Scientific, San Jose, CA, USA) coupled with Orbitrap Q Exactive Plus (Thermo Fisher Scientific, San Jose, CA, USA) was used to analyze the metabolites. The separation of metabolites using UHPLC conditions was as follows: mobile phase A contained $0.1 \%(v / v)$ Formic acid and $5 \mathrm{mM}$ ammonium acetate at pH 9.0 in water, while mobile phase B comprised $99.7 \%$ methanol. The extraction solutions were injected into the reverse phase column, Hypersil GoldColumn $(100 \times 2.1 \mathrm{~mm}$ with $1.9 \mu \mathrm{m}$ particle size). The injection volume was $10 \mu$, the flow rate was $0.25 \mathrm{~mL} / \mathrm{min}$, and the column temperature was $20{ }^{\circ} \mathrm{C}$. The gradient conditions were as follows: $0 \mathrm{~min}, 98 \%$ $\mathrm{A}$ and $2 \% \mathrm{~B} ; 1.5 \mathrm{~min}, 98 \% \mathrm{~A}$ and $2 \% \mathrm{~B} ; 12 \mathrm{~min}, 0 \% \mathrm{~A}$ and $100 \% \mathrm{~B} ; 14 \mathrm{~min} 0 \% \mathrm{~A}$ and $100 \%$ B; $14.1 \mathrm{~min} 98 \%$ A and $2 \%$ B; 17 min $98 \%$ A and 2\% B. The Orbitrap Q Exactive Plus mass spectrometer was operated in positive polarity mode with $3.2 \mathrm{kV}$ of spray voltage. The capillary temperature was $320^{\circ} \mathrm{C}$; the temperature of the autosampler was $10^{\circ} \mathrm{C} ; 35 \mathrm{arb}$ and 10 arb were the sheath gas flow rate and auxiliary gas flow rate, respectively [53].

\subsection{Analysis of Metabolome Data}

Compound discoverer v.3.1 (CD 3.1, Thermo Scientific, San Jose, CA, USA) was used for mass spectrometry data acquisition [53]. The settings were as follows: 5 ppm mass tolerance, 30 signal of intensity tolerance, and 1,000,000 of minimum peak intensity [54]. The annotations of metabolites were generated using ChemSpider (http:/ /www.chemspider.com/) (accessed date: 29 January 2021) database, which consisted of integrating data from the Aracyc, Biocyc, KEGG pathways, mzVault, and mzCloud databases. Differentially accumulated metabolites by chemical treatments were selected with $p$-value $<0.05$, fold change $\geq 1.5$ 
and $\leq 0.667$. Volcano plots were generated to show the distribution of differentially accumulated metabolites.

Supplementary Materials: The following are available online at https:/ / www.mdpi.com/1422-0 067/22/6/2968/s1, Figure S1: Incidence rates of FHB disease in wheat cultivar USU-Apogee from 4 to 28 dpi, Figure S2: FHB disease of wheat spikes was suppressed by NMN and NIM pretreatment at 12 dpi, Figure S3: NIM and NMN effectively suppressed FHB disease in wheat spikes cultivar Haruyutaka at $5 \mathrm{dpi}$, Figure S4: FHB disease was suppressed by NIM- or NMN-pretreatment in wheat cultivar Harukirari at $10 \mathrm{dpi}$.

Author Contributions: Conceptualization, T.Y., M.K. and T.N.; methodology, M.N., Y.M. and T.N.; investigation, Y.S., M.N. and Y.M.; data curation, Y.S. and M.N.; writing-original draft preparation, Y.S.; writing-review and editing, T.N.; project administration, M.K.; funding acquisition, M.K. and T.N. All authors have read and agreed to the published version of the manuscript.

Funding: This research was supported by grants from the Project of the NARO Bio-oriented Technology Research Advancement Institution (Research program on development of innovative technology, no. 28007A and 28007AB). This work was also supported by Research for Promoting Technological Seeds (no. 07-070) and by the Adaptable and Seamless Technology Transfer Program through Target-driven R\&D (AS232Z02753E and AS242Z03390N) from the Japan Science and Technology Agency (JST). This research was supported partly by Scientific Research (no. 23580060, 26450053, 15H05780, and 18K05642) from the Ministry of Education, Culture, Sports, Science and Technology of Japan. This research was partially supported by Indonesia Endowment Fund for Education (LPDP) and Ministry of Research, Technology, and Higher Education of Indonesia (BUDI-LN Scholarship, FR2732019174118).

Institutional Review Board Statement: Not applicable.

Informed Consent Statement: Not applicable.

Data Availability Statement: The data presented in this study are available online within this article or supplementary materials.

Conflicts of Interest: The authors declare no conflict of interest.

\section{References}

1. Andersen, E.J.; Ali, S.; Byamukama, E.; Yen, Y.; Nepal, M.P. Disease Resistance Mechanisms in Plants. Genes 2018, 9, 399. [CrossRef] [PubMed]

2. Ahuja, I.; Kissen, R.; Bones, A.M. Phytoalexins in defense against pathogens. Trends Plant Sci. 2012, 17, 73-90. [CrossRef]

3. Bolanos-Carriel, C.; Wegulo, S.N.; Baenziger, P.S.; Funnell-Harris, D.; Hallen-Adams, H.E.; Eskridge, K.M. Effects of fungicide chemical class, fungicide application timing, and environment on Fusarium head blight in winter wheat. Eur. J. Plant Pathol. 2020, 158, 1-13. [CrossRef]

4. D'Mello, J.F.; Macdonald, A.M.; Postel, D.; Dijksma, W.T.; Dujardin, A.; Placinta, C.M. Pesticide Use and Mycotoxin Production in Fusarium and Aspergillus Phytopathogens. Eur. J. Plant Pathol. 1998, 104, 741-751. [CrossRef]

5. Sabarwal, A.; Kumar, K.; Singh, R.P. Hazardous effects of chemical pesticides on human health-Cancer and other associated disorders. Environ. Toxicol. Pharmacol. 2018, 63, 103-114. [CrossRef]

6. Sevastos, A.; Kalampokis, I.; Panagiotopoulou, A.; Pelecanou, M.; Aliferis, K. Implication of Fusarium graminearum primary metabolism in its resistance to benzimidazole fungicides as revealed by $1 \mathrm{H}$ NMR metabolomics. Pestic. Biochem. Physiol. 2018, 148, 50-61. [CrossRef] [PubMed]

7. Iwai, T.; Seo, S.; Mitsuhara, I.; Ohashi, Y. Probenazole-Induced Accumulation of Salicylic Acid Confers Resistance to Magnaporthe grisea in Adult Rice Plants. Plant Cell Physiol. 2007, 48, 915-924. [CrossRef] [PubMed]

8. Yoshioka, K.; Nakashita, H.; Klessig, D.F.; Yamaguchi, I. Probenazole Induces Systemic Acquired Resistance in Arabidopsis with a Novel Type of Action. Plant J. 2001, 25, 149-157. [CrossRef] [PubMed]

9. Vernooij, B.; Friedrich, L.; Goy, P.A.; Staub, T.; Kessmann, H.; Ryals, J. 2,6-Dichloroisonicotinic Acid-Induced Resistance to Pathogens without the Accumulation of Salicylic Acid. Mol. Plant-Microbe Interact. 1995, 8, 228-234. [CrossRef]

10. Friedrich, L.; Lawton, K.; Ruess, W.; Masner, P.; Specker, N.; Rella, M.G.; Meier, B.; Dincher, S.; Staub, T.; Uknes, S.; et al. A benzothiadiazole derivative induces systemic acquired resistance in tobacco. Plant J. 1996, 10, 61-70. [CrossRef]

11. Haque, A.; Wang, Y.; Shen, Z.; Li, X.; Saleemi, M.K.; He, C. Mycotoxin contamination and control strategy in human, domestic animal and poultry: A review. Microb. Pathog. 2020, 142, 104095. [CrossRef]

12. Zain, M.E. Impact of mycotoxins on humans and animals. J. Saudi Chem. Soc. 2011, 15, 129-144. [CrossRef] 
13. Nishiuchi, T. Plant Responses to Fusarium Metabolites. In Fusarium: Genomics, Molecular and Cellular Biology; Brown, D.W., Proctor, R.H., Eds.; Caister Academic Press: Norfolk, UK, 2013; pp. 165-291.

14. Kokkonen, M.; Ojala, L.; Parikka, P.; Jestoi, M. Mycotoxin production of selected Fusarium species at different culture conditions. Int. J. Food Microbiol. 2010, 143, 17-25. [CrossRef]

15. McMullen, M.; Bergstrom, G.; De Wolf, E.; Dill-Macky, R.; Hershman, D.; Shaner, G.; Van Sanford, D. A Uniunified Effort to Fight an Enemy of Wheat and Barley: Fusarium Head Blight. Plant Dis. 2012, 96, 1712-1728. [CrossRef]

16. Giraud, F.; Pasquali, M.; El Jarroudi, M.; Cocco, M.; Delfosse, P.; Hoffmann, L.; Bohn, T. Timely Fungicide Application: A Strategy to Minimize Fusarium Head Blight and Associated Mycotoxin Production in Winter Wheats. J. Plant Pathol. 2011, 93, 16-19. [CrossRef]

17. Miwa, A.; Sawada, Y.; Tamaoki, D.; Hirai, M.Y.; Kimura, M.; Sato, K.; Nishiuchi, T. Nicotinamide mononucleotide and related metabolites induce disease resistance against fungal phytopathogens in Arabidopsis and barley. Sci. Rep. 2017, 7, 6389. [CrossRef]

18. Maeda, K.; Nakajima, Y.; Tanahashi, Y.; Kitou, Y.; Miwa, A.; Kanamaru, K.; Kobayashi, T.; Nishiuchi, T.; Kimura, M. L-Threonine and its analogue added to autoclaved solid medium suppress trichothecene production by Fusarium graminearum. Arch. Microbiol. 2017, 199, 945-952. [CrossRef]

19. Wang, C.; Zhou, M.; Zhang, X.; Yao, J.; Zhang, Y.; Mou, Z. A Lectin Receptor Kinase as a Potential Sensor for Extracellular Nicotinamide Adenine Dinucleotide in Arabidopsis thaliana. Elife 2017, 6, 1-23. [CrossRef] [PubMed]

20. Zhonglin, M. Extracellular Pyridine Nucleotides as Immune Elicitors in Arabidopsis. Plant Signal. Behav. 2017, 12 , e1388977.

21. Zhang, X.; Mou, Z. Extracellular Pyridine Nucleotides Induce PR Gene Expression and Disease Resistance in Arabidopsis. Plant J. 2009, 57, 302-312. [CrossRef] [PubMed]

22. Berglund, T.; Wallström, A.; Nguyen, T.V.; Laurell, C.; Ohlsson, A.B. Nicotinamide; Antioxidative and DNA Hypomethylation Effects in Plant Cells. Plant Physiol. Biochem. 2017, 118, 551-560. [CrossRef] [PubMed]

23. Mackintosh, C.A.; Garvin, D.F.; Radmer, L.E.; Heinen, S.J.; Muehlbauer, G.J. A model wheat cultivar for transformation to improve resistance to Fusarium Head Blight. Plant Cell Rep. 2005, 25, 313-319. [CrossRef]

24. He, X.; Dreisigacker, S.; Singh, R.P.; Singh, P.K. Genetics for low correlation between Fusarium head blight disease and deoxynivalenol (DON) content in a bread wheat mapping population. Theor. Appl. Genet. 2019, 132, 2401-2411. [CrossRef]

25. Nakamichi, K.; Sato, M.; Yoshimura, Y.; Kobayashi, S.; Nishimura, T.; Ikenaga, M.; Ashinaga, N.; Araki, K.; Yanagisawa, A.; Kon, T.; et al. A New Spring Wheat Variety "Harukirari". Bull. Hokkaido Res. Organ. Agric. Exp. Station. 2011, 95, 25-37. (In Japanese)

26. Nishio, Z.; Takata, K.; Ito, M.; Tanio, M.; Tabiki, T.; Yamauchi, H.; Ban, T. Deoxynivalenol Distribution in Flour and Bran of Spring Wheat Lines with Different Levels of Fusarium Head Blight Resistance. Plant Dis. 2010, 94, 335-338. [CrossRef]

27. Osbourn, A. Saponins and Plant Defence-a Soap Story. Trend Plant Sci. 1996, 1, 4-9. [CrossRef]

28. Thawabteh, A.; Juma, S.; Bader, M.; Karaman, D.; Scrano, L.; Bufo, S.A.; Karaman, R. The Biological Activity of Natural Alkaloids against Herbivores, Cancerous Cells and Pathogens. Toxins 2019, 11, 656. [CrossRef] [PubMed]

29. Lemaitre-Guillier, C.; Fontaine, F.; Roullier-Gall, C.; Harir, M.; Magnin-Robert, M.; Clément, C.; Trouvelot, S.; Gougeon, R.D.; Schmitt-Kopplin, P.; Adrian, M. Cultivar- and Wood Area-Dependent Metabolomic Fingerprints of Grapevine Infected by Botryosphaeria dieback. Phytopathology 2020, 110, 1821-1837. [CrossRef]

30. Peng, F.-J.; Ying, G.-G.; Liu, Y.-S.; Su, H.-C.; He, L.-Y. Joint antibacterial activity of soil-adsorbed antibiotics trimethoprim and sulfamethazine. Sci. Total. Environ. 2015, 506-507, 58-65. [CrossRef]

31. Nakagawa, S.; Tachrim, Z.P.; Kurokawa, N.; Ohashi, F.; Sakihama, Y.; Suzuki, T.; Hashidoko, Y.; Hashimoto, M. pH Stability and Antioxidant Power of CycloDOPA and Its Derivatives. Molecules 2018, 23, 1943. [CrossRef]

32. Ahmad, S.; Veyrat, N.; Gordon-Weeks, R.; Zhang, Y.; Martin, J.; Smart, L.; Glauser, G.; Erb, M.; Flors, V.; Frey, M.; et al. Benzoxazinoid Metabolites Regulate Innate Immunity against Aphids and Fungi in Maize. Plant Physiol. 2011, 157, 317-327. [CrossRef]

33. Ojika, M.; Suzuki, Y.; Tsukamoto, A.; Sakagami, Y.; Fudou, R.; Yoshimura, T.; Yamanaka, S. Cystothiazoles A and B, new bithiazole-type antibiotics from the myxobacterium Cystobacter fuscus. J. Antibiot. 1998, 51, 275-281. [CrossRef] [PubMed]

34. Aucique-Pérez, C.E.; Resende, R.S.; Neto, L.B.C.; Dornelas, F.; DaMatta, F.M.; Rodrigues, F. Ávila Picolinic acid spray stimulates the antioxidative metabolism and minimizes impairments on photosynthesis on wheat leaves infected by Pyricularia oryzae. Physiol. Plant. 2019, 167, 628-644. [CrossRef] [PubMed]

35. Mogg, C.; Bonner, C.; Wang, L.; Schernthaner, J.; Smith, M.; Desveaux, D.; Subramaniam, R. Genomic Identification of the TOR Signaling Pathway as a Target of the Plant Alkaloid Antofine in the Phytopathogen Fusarium graminearum. mBio 2019, 10, e00792-19. [CrossRef] [PubMed]

36. Tyihák, E.; Sarhan, A.R.T.; Cong, N.T.; Barna, B.; Király, Z. The level of trigonelline and other quaternary ammonium compounds in tomato leaves in ratio to the changing nitrogen supply. Plant Soil 1988, 109, 285-287. [CrossRef]

37. Kraska, T.; Schönbeck, F. About Changes in the Chromatin Structure after Resistance Induction in Hordeum vulgare L. J. Phytopathol. 1993, 137, 10-14. [CrossRef]

38. Sittiwet, C.; Puangpronpitag, D. Anti-Bacterial Activity of Cryptolepis buchanani Aqueous Extract. Int. J. Biol. Chem. 2009, 3, 90-94. [CrossRef]

39. Supriyono, A.; Schwarz, B.; Wray, V.; Witte, L.; Müller, W.E.G.; Van Soest, R.; Sumaryono, W.; Proksch, P. Bioactive Alkaloids from the Tropical Marine Sponge Axinella carteri. Z. Nat. C 1995, 50, 669-674. [CrossRef] 
40. Berglund, T. Nicotinamide, a missing link in the early stress response in eukaryotic cells: A hypothesis with special reference to oxidative stress in plants. FEBS Lett. 1994, 351, 145-149. [CrossRef]

41. Espinas, N.A.; Saze, H.; Saijo, Y. Epigenetic Control of Defense Signaling and Priming in Plants. Front. Plant Sci. $2016,7,1201$. [CrossRef]

42. Zhang, H.; Deng, X.; Miki, D.; Cutler, S.; La, H.; Hou, Y.-J.; Oh, J.; Zhu, J.-K. Sulfamethazine Suppresses Epigenetic Silencing in Arabidopsis by Impairing Folate Synthesis. Plant Cell 2012, 24, 1230-1241. [CrossRef]

43. Fuentes-Contreras, E.; Niemeyer, H.M. DIMBOA Glucoside, a Wheat Chemical Defense, Affects Host Acceptance and Suitability of Sitobion avenae to the Cereal Aphid Parasitoid Aphidius rhopalosiphi. J. Chem. Ecol. 1998, 24, 371-381. [CrossRef]

44. Zhang, C.; Li, J.; Li, S.; Ma, C.; Liu, H.; Wang, L.; Qi, J.; Wu, J. ZmMPK6 and ethylene signalling negatively regulate the accumulation of anti-insect metabolites DIMBOA and DIMBOA-Glc in maize inbred line A188. New Phytol. 2021, 229, $2273-2287$. [CrossRef] [PubMed]

45. Etzerodt, T.; Maeda, K.; Nakajima, Y.; Laursen, B.; Fomsgaard, I.S.; Kimura, M. 2,4-Dihydroxy-7-methoxy-2 H -1,4-benzoxazin-3(4 $\mathrm{H}$ )-one (DIMBOA) inhibits trichothecene production by Fusarium graminearum through suppression of Tri6 expression. Int. J. Food Microbiol. 2015, 214, 123-128. [CrossRef] [PubMed]

46. Alvarez, F.J.; Douglas, L.M.; Konopka, J.B. Sterol-Rich Plasma Membrane Domains in Fungi. Eukaryot. Cell 2007, 6, 755-763. [CrossRef]

47. Forrer, H.-R.; Musa, T.; Schwab, F.; Jenny, E.; Bucheli, T.D.; Wettstein, F.E.; Vogelgsang, S. Fusarium Head Blight Control and Prevention of Mycotoxin Contamination in Wheat with Botanicals and Tannic Acid. Toxins 2014, 6, 830-849. [CrossRef]

48. Francesconi, S.; Steiner, B.; Buerstmayr, H.; Lemmens, M.; Sulyok, M.; Balestra, G.M. Chitosan Hydrochloride Decreases Fusarium graminearum Growth and Virulence and Boosts Growth, Development and Systemic Acquired Resistance in Two Durum Wheat Genotypes. Molecules 2020, 25, 4752. [CrossRef]

49. Maeda, K.; Nakajima, Y.; Tanahashi, Y.; Kosaki, T.; Kitou, Y.; Kanamaru, K.; Kobayashi, T.; Nishiuchi, T.; Kimura, M. Characterization of the acivicin effects on trichothecene production by Fusarium graminearum species complex. J. Gen. Appl. Microbiol. 2016, 62, 272-276. [CrossRef]

50. Wahibah, N.N.; Tsutsui, T.; Tamaoki, D.; Sato, K.; Nishiuchi, T. Expression of barley Glutathione S-Transferase13 gene reduces accumulation of reactive oxygen species by trichothecenes and paraquat in Arabidopsis plants. Plant Biotechnol. 2018, 35, 71-79. [CrossRef]

51. Siou, D.; Gelisse, S.; Laval, V.; Repincay, C.; Canalès, R.; Suffert, F.; Lannou, C. Effect of wheat spike infection timing on fusarium head blight development and mycotoxin accumulation. Plant Pathol. 2014, 63, 390-399. [CrossRef]

52. Paolacci, A.R.; Tanzarella, O.A.; Porceddu, E.; Ciaffi, M. Identification and validation of reference genes for quantitative RT-PCR normalization in wheat. BMC Mol. Biol. 2009, 10, 11. [CrossRef] [PubMed]

53. Liu, Y.; Kong, Z.; Liu, J.; Zhang, P.; Wang, Q.; Huan, X.; Li, L.; Qin, P. Non-Targeted Metabolomics of Quinoa Seed Filling Period Based on Liquid Chromatography-Mass Spectrometry. Food Res. Int. 2020, 137, 109743. [CrossRef] [PubMed]

54. Scarpone, R.; Rosato, R.; Chiumiento, F.; Cipolletti, C.; Sergi, M.; Compagnone, D. Preliminary Study to Develop an Alternative Method for the Non-targeted Determination of Xenobiotics in Food by Means of Ultra High Performance Liquid Chromatography Coupled to High Resolution and Accuracy Mass Spectrometry. Food Anal. Methods 2020, 13, 1099-1110. [CrossRef] 